\title{
Measurement of Multimode Fiber Bandwidth by the Fourth-Order Spectra of Amplified Spontaneous Emission
}

\author{
Sucbei Moon ${ }^{1}$ and Dug Young $\mathrm{Kim}^{2} *$ \\ ${ }^{1}$ Department of Physics, Kookmin University, Seoul 136-702, Korea \\ ${ }^{2}$ Department of Physics, Yonsei University, Seoul 120-749, Korea
}

(Received November 26, 2013 : revised January 21, 2014 : accepted February 3, 2014)

\begin{abstract}
We present a novel bandwidth measurement scheme for multimode optical fibers. Amplified spontaneous emission (ASE) radiation was utilized for a source of intrinsically modulated light with a wide modulation bandwidth. In our measurement scheme, the continuous-wave (CW) ASE light that passed through a multimode fiber (MMF) under test was analyzed by the fourth-order power with a high-speed photodetector and an electric spectrum analyzer. The modulation transfer function of the multimode fiber could be directly measured with the photoelectric spectra in the modulation frequency domain. The measurement result of our method was experimentally compared to that of the conventional measurement scheme based on the impulse response measurement. It has been found that our scheme provides a stable measurement means of MMF characterization that is suitable for the field testing due to the simplicity of the system.

Keywords: Multimode fiber, Bandwidth measurement, Amplified spontaneous emission, Spectral intensity interferometry

OCIS codes : (060.2270) Fiber characterization; (060.2300) Fiber measurements; (120.4640) Optical instruments
\end{abstract}

\section{INTRODUCTION}

The multimode fiber (MMF) technology is widely used in short-haul optical networks such as a local-area network (LAN) or a peripheral network $[1,2]$. In those optical communication systems, the typical length of the MMF links is a few hundreds of meters or shorter with usual bit rates of 1 to $10 \mathrm{Gbps}$. The MMF class has relative advantages of larger tolerances in fiber connection and the availability of the high-speed vertical cavity surface emitting lasers (VCSELs) that are better suited to the MMF [1-3]. Accurate evaluation of the MMF performance is indispensible in designing such a system. In contrast to the single-mode fibers (SMFs) where the group-velocity dispersion (GVD) limits the maximum data rates, the chromatic dispersion plays little role in the MMF. It has a plurality of guiding modes that propagate at different group velocities with characteristic intermodal delays [4-6]. The link performance of the MMF can be properly described by the modulation bandwidth which is the frequency-domain measure of the intermodal dispersion. For this, several methods of the MMF bandwidth measurement have been developed and widely used in evaluation of the manufactured MMF cables as the standard methods [5, 6]. It is worth stressing that each MMF cable even from a single preform needs to be evaluated in MMF bandwidth for reliable and predictable transmission performance [2]. By the nature of the MMF, a slight deviation of the refractive index profile can critically affect the modal dispersion in a sensitive manner.

It has been found that a number of factors can affect the actual bandwidth performance of the installed MMF link, not to mention the non-uniformity of the index profile along the fiber axis $[4,6,7]$. The light launch condition, operation wavelength, quality of splices or connectors, fiber bends and polarization states may considerably influence the actual bandwidth of the MMF link because they determine or change the mode power distribution and, consequently, influence the effective intermodal delays. This brings the needs for evaluating the bandwidth performance of the MMF links after the fiber

\footnotetext{
*Corresponding author: dykim1@yonsei.ac.kr

Color versions of one or more of the figures in this paper are available online.
} 
has been installed in the fields. Particularly, field testing is highly demanded in upgrading the installed fiber networks for higher speeds when the information on the old MMF is insufficient. However, the current technologies used for the MMF bandwidth measurements do not provide desired simplicity and can rarely be used in field testing. Furthermore, as the MMF-based networks have evolved to Gigabit or 10-Gigabit systems, the MMF links in the fields usually have very wide bandwidths above several GHz's or higher [1]. It is technically more challenging than measuring long sections of manufactured MMF cables. A new bandwidth measurement method is demanded with a simpler system configuration and a wider effective measurement bandwidth, definitely above $10 \mathrm{GHz}$.

In this report, we present an MMF bandwidth measurement scheme that significantly simplifies the system configuration and the related maintenance issues. Our scheme uses a low-cost light source of amplified spontaneous emission (ASE) and a popular radio-frequency (RF) measurement instrument of the electric spectrum analyzer (ESA). Our scheme utilizes the ASE light that intrinsically exhibits random fluctuations of intensity which are determined by the optical spectrum. Due to the wide bandwidth along with the nearly uniform spectral characteristic of the ASE, the transmission characteristic of the MMF can be analyzed with ease by obtaining the photoelectric spectrum detected at the output of the MMF under test. Used in the MMF bandwidth measurement, the practically unlimited bandwidth of the ASE light minimizes the needs for careful calibrations of the light source and the concerns associated with the unwanted deviations. In this work, we successfully demonstrated the MMF bandwidth measurement and compared the result to that of the conventional scheme based on a short-pulse laser. It has been found that our scheme of the ASE-based MMF bandwidth measurement provides a simple and powerful test tool with a good measurement reliability, which will be useful in the field testing of MMF bandwidth as well as for a generic evaluation technique of the MMF cables.

\section{MMF BANDWIDTH MEASUREMENT}

The MMF bandwidth quantifies the modal dispersion that the lightwave signal experiences due to the intermodal delays of the mode-dependent group velocities [5]. Neglecting the chromatic dispersion effect that occurs for each mode, the transmitted signal is broadened in accordance with the modal characteristics. A signal input at an end of MMF is denoted by $f_{\text {in }}(t)$ as a time-domain function of optical power. Then, the output signal obtained at the other end, denoted by $f_{\text {out }}(t)$, can be expressed by a sum of delayed Dirac delta functions of time $t$, i.e., a sum of $\delta\left(t-\tau_{n}\right)$, as

$$
f_{\text {out }}(t)=\sum_{j}\left\{f_{\text {in }}(t) \otimes \delta\left(t-\tau_{n}\right) \cdot P_{n}\right\}
$$

where $n$ is the modal index of integer, $\tau_{n}$ is the group delay, and $P_{n}$ is the modal power for each mode of $n$, respectively. Here, $\otimes$ denotes an integral convolution. The power-spread model of Eq. (1) is an approximate way of understanding the MMF dispersion which is suitable for typical MMFs where the field-sensitive phenomena are averaged out for a large number of modes. Eq. (1) can be rewritten in a simple form as

$$
f_{\text {out }}(t)=f_{\text {in }}(t) \otimes h(t)
$$

where $h(t)$ is the characteristic function of the MMF, called as the impulse response in the linear system analysis. The impulse response is, then, a sum of the power transfer with modal delays as expressed by

$$
h(t)=\sum_{n} P_{n} \cdot \delta\left(t-\tau_{n}\right)
$$

with $P_{n}$ 's of the normalized sum. The Fourier transform of Eq. (2) gives a simple algebraic formula as

$$
F_{\text {out }}(\omega)=F_{\text {in }}(\omega) \cdot H(\omega)
$$

where $F_{\text {out }}, F_{\text {in }}$ and $H$ are the Fourier conjugates of $f_{\text {out }}, f_{\text {in }}$ and $h$ in frequency $\omega$, respectively. The transfer function of $H(\omega)$ is the Fourier transform of the impulse response, $h(t)$, and it determines the bandwidth of MMF. It is typically estimated by the frequency at which the amplitude of $H(\omega)$ decreases to $-3 \mathrm{~dB}(50 \%)$ in respect to $H(0)$. This modulation bandwidth must be distinguished from an optical bandwidth. Notice that the description of Eq. (1) through Eq. (4) may include the effects of intermodal coupling and mode-dependent loss that the installed MMF may contain in the middle of the link. Those effects can be approximately characterized as exchange or decrease of the modal powers in the power-spread model of MMF.

The bandwidth of an MMF partially depends on the light launching condition that determines how the modes are excited. In Eq. (3), it can be represented by the mode power distribution of $P_{j}$. As a neutral launch condition, the classic method of the MMF bandwidth measurement utilizes the over-filled launch (OFL) in which the light field is incident with an excessively high numerical aperture (NA) with a low degree of spatial coherence $[5,6]$. Then, it can fully fill the angular and spatial components that excite virtually all the modes of the MMF with equal amounts of power as this can be described by a uniform value of $P_{n}$ in Eq. (3). This OFL condition can be accomplished with ease for the case of incoherent light sources such as a light emitting diode (LED). Otherwise, especially with a laser source, a mode scrambler or mode mixer can be used, which strongly couples the guiding modes with intentionally applied local perturbations such as fiber bends. A near equilibrium of the mode power distribution 
can be established by this manner even with a highly coherent light source.

Based on the theory of modulation transfer, the MMF bandwidth can be analyzed in either time domain or frequency domain by utilizing a light source of a wide modulation bandwidth [5]. The most popular scheme of the MMF bandwidth measurement is to obtain the impulse response in time with impulse-like short pulses. In an alternative approach, the transfer function can be directly obtained in frequency domain by using a light source modulated sinusoidally with a variable frequency. In both methods, the technical difficulty may arise in the source calibration because the modulation spectrum of the source is not uniform over the whole measurement frequency range and its bandwidth is finite. The modulation bandwidth of those light sources such as a gain-switched diode laser or an intensity modulator-equipped laser gives a few tens of GHz's at best. Thus, the reliability of the measured result crucially depends on the stability of the modulated light source and the calibration accuracy, especially when the measurement range is wider than $10 \mathrm{GHz}$.

In this research on the MMF bandwidth measurement, we utilized an ASE light in continuous-wave (CW) operation instead of using a modulated light source. The ASE light consists of continuously distributed spectral components of random phases which produce cross-beating products also known as the relative intensity noise [8]. The modulation spectrum after the down-conversion process of a squarelaw detector can be found by the autocorrelation function of the optical spectrum. Hence, the modulation bandwidth of the ASE is as wide as the optical bandwidth of the spectrum. A very stable modulation characteristic can be obtained when the spectrum is determined by a passive element of an optical filter. Then, the modulation transfer function of an MMF can be obtained by comparing the modulation spectra measured at the different ports of the MMF under test.

The principle of our measurement scheme may need a theoretical justification. The intrinsic intensity modulation of the ASE fields has a different nature from the modulated lightwaves such as a pulsed laser or externally modulated light. It is not automatically accepted that the intensity-modulated contents of the ASE are temporally dispersed in the same way as those of the modulated lightwave signals. A suitable description of the ASE light fields that pass through an MMF is obtained from the spectral intensity interferometer [8]. Applying this theory, the MMF can be understood as an interferometer of multiple light paths like a multi-arm Mach-Zehnder interferometer (MZI). Each path of a guiding mode or a coupled mode provides a characteristic delay. Figure 1 shows the schematic diagrams that illustrate the MMF with an ASE source and a photodetector (a), the corresponding multi-arm MZI (b), and the contents of the ASE light at the source part (c), respectively. Both of the MMF and the MZI give the multi-path by which the light fields of the ASE are dispersed with time delays of $\tau_{n}$ 's. The modulation spectrum of the ASE is an integration of the two-component beatings and makes a random fluctuation of intensity as explained by Fig. 1(c). Our bandwidth measurement system measures the intermodal delays from the change of the intensity spectra induced by the multi-path of the MMF.

The MMF multi-path inevitably brings an uncontrolled variation of the polarization states and path length fluctuations which may sensitively affect the output fields in conjunction with mode coupling or intermodal interference. The spectral intensity interferometry suggests that, regardless of the field-sensitive process, the fourth-order power spectrum of the resultant field at the multi-path output conveys only the intensity-involved information [8]. This convenience is obtained by a narrow bandwidth of the ASE which must be less than 1/4 of the center frequency of the optical spectrum. The usual ASE light sources surely fulfill this narrow-band condition. Therefore, the photoelectric power spectrum of a photodiode, which is the forth power of the ASE field, is independent of the field-sensitive interference that occurs in the MMF.

Interferometric measurement of the intermodal delays or the modal dispersion of an MMF is not a completely new concept. It has been revealed that the optical frequencydomain reflectometry or the spectral interferometry can measure the modal characteristic in an interferometric configuration [9-11]. However, those second-order interferometers sense the field-sensitive interference so that they are naturally vulnerable to the phase and polarization variations of the modes. Despite some attractive features of the second-order interferometry, the result is hardly translated to the MMF bandwidth without a priori knowledge on the MMF modes. Our ASE-based measurement method that utilizes the fourth-order interferometry is exclusively sensitive to the intensity contents that can accurately evaluate the bandwidth performance of the intensity-modulated communication link.

(a)
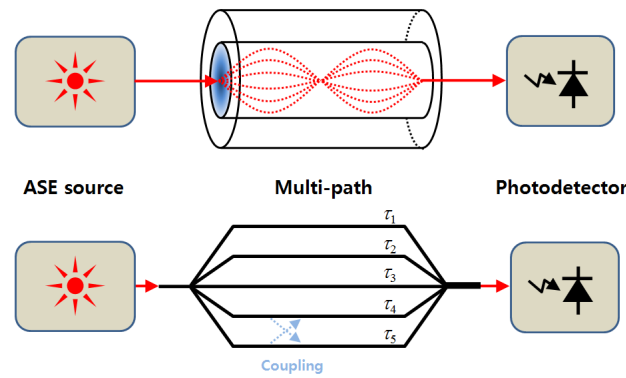

(c)
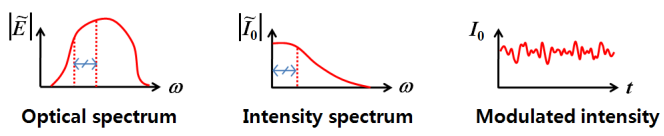

FIG. 1. Schematic diagrams that illustrate the MMF with an ASE source and a photodetector (a), the corresponding multi-arm MZI (b), and the contents of the ASE light at the source part (c), respectively. 
A more detailed description on our ASE-based measurement method is presented here to clarify the operation principle. Any lightwave of $E(t)$ can be expressed with the center frequency of the spectrum $\omega_{0}$ as

$$
E(t)=A(t) \cdot e^{i \omega_{0} t}
$$

where $A(t)$ is the complex amplitude of the wave field. The ASE light has a broad spectrum centered at $\omega_{0}$ with a random phase at each frequency. The intensity, denoted by $I(t)$, is fluctuating as a consequence. The spectrum of the intensity is found by the Fourier transform of $I(t)$. Denoting the Fourier transform with $\mathbf{F}\{\cdots\}$, the intensity spectrum at the source, $I_{0}(t)$, has its intensity spectrum as

$$
\tilde{I}_{0}(\omega) \equiv \mathbf{F}\left\{E^{*} \cdot E\right\}=\tilde{A}^{*}(-\omega) \otimes \tilde{A}(\omega)
$$

where $\widetilde{I}_{0}$ and $\widetilde{A}$ are the Fourier conjugates of $I_{0}$ and $A$, respectively. Eq. (6) suggests that the intensity spectrum is the spectral autocorrelation function of the amplitude spectrum. This ASE light goes through the multi-path given by the MMF. From the result derived in Ref. 8, the fourth-order spectrum $S^{(4)}$ in modulation frequency domain is found under the narrow-band condition as

$$
S^{(4)}(\omega) \equiv|\tilde{I}(\omega)|^{2}=\sum_{n} a_{n}^{2}\left|\tilde{I}_{0}\right|^{2}+\sum_{n>m} 2 a_{n} a_{m}\left|\tilde{I}_{0}\right|^{2} \cos \omega \tau_{n m}
$$

when detected at the MMF output. Here, $n$ and $m$ are the mode indices, and the spatially averaged optical intensity of a mode of $n$ is denoted by $a_{n} \cdot I_{0}(t)$ with a positive real coefficient of $a_{n}$. The relative delay of $\tau_{n m}$ is defined by the temporal difference of $\tau_{n}-\tau_{m}$ between the two modes. It is assumed, in Eq. (7), that the intensity spectrum at the output is the same as $\widetilde{I}_{0}$ for each mode, which implies neglecting the GVD effect for the MMF.

From Eq. (3), the transfer function of the MMF is derived to be a sum of the transformed delta functions as

$$
H(\omega)=\sum_{n} P_{n} e^{i \omega \tau_{n}}
$$

by the property of the Fourier transform with the mode index $n$. Its amplitude is, thus, found by

$$
|H(\omega)|^{2}=\left(\sum_{n} P_{n} e^{-i \omega \tau_{n}}\right)\left(\sum_{m} P_{m} e^{i \omega \tau_{m}}\right)=\sum_{n} a_{n}^{2}+\sum_{n>m} 2 a_{n} a_{m} \cos \omega \tau_{n m}
$$

when $P_{n}=a_{n}$ is taken for simplicity. Therefore, the squared absolute transfer function of Eq. (9) is proportional to the fourth-order spectrum of $S^{(4)}$ given in Eq. (7). The amplitude of the transfer function is obtained easily from the experimental data as

$$
|H(\omega)|=\sqrt{\frac{S^{(4)}(\omega)}{\left|\tilde{I}_{0}(\omega)\right|^{2}}}
$$

in our ASE-based measurement method. The power of $\widetilde{I}_{0}$ can be obtained in a separate measurement performed with no MMF under test in place.

\section{EXPERIMENT}

In this investigation, we constructed an ASE-based MMF bandwidth measurement system with an ASE light source and an electric spectrum analyzer. The measurement result was compared to that of the conventional method based on a short-pulse laser. Figure 2 shows the schematic diagrams of the filtered-ASE source (a), our ASE-based MMF bandwidth measurement system (b), and that of the pulse-based measurement system that we used for comparison. As shown in Fig. 2(a), a semiconductor optical amplifier (SOA) produced ASE light and also re-amplified the ASE back-reflected by a fiber-optic reflector for a higher intensity. After an optical isolator, a band-pass filter (BPF) was installed to reduce the optical bandwidth to $20 \mathrm{~nm}$, centered at $1,310 \mathrm{~nm}$ in wavelength. The intensity was adjusted with an optical attenuator. All the fiber-optic components in the source part used single-mode fiber for interconnection.

In our measurement system described in Fig. 2(b), the ASE light fed by the source part went through a conditioning MMF for mode scrambling. A series of perturbations including bends and offset splices were applied to the conditioning MMF of a high-NA step-index fiber. The mode-scrambled light was launched to a fiber under test (FUT), a graded-index MMF designed for optical communications, through a fiber connector. A photoreceiver connected to the FUT converted the received intensity signal to the photocurrent and fed it to an electric spectrum analyzer

(a)

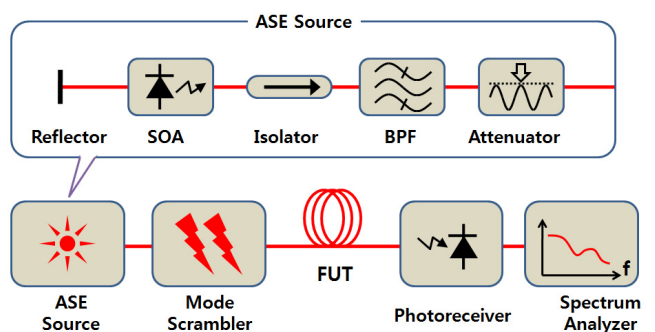

(c)

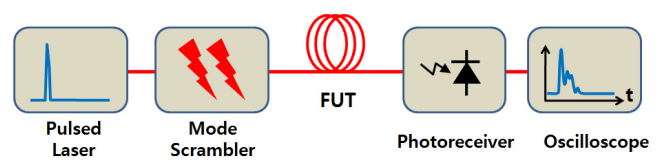

FIG. 2. Schematic diagrams of the filtered-ASE source (a), our ASE-based MMF bandwidth measurement system (b), and that of the pulse-based measurement system that we used for comparison. 
(E4440A, Agilent Technologies Inc.) that acquired the power spectrum of the photocurrent. The photoreceiver used in the system equipped a high-speed photodiode and an internal amplifier with a combined bandwidth of $11 \mathrm{GHz}$. For comparison, the conventional impulse response measurement system was constructed by using the same mode scrambler and photoreceiver. A gain-switched laser diode of distributed feedback (DFB) laser was used for the short-pulse source while a wideband sampling oscilloscope (DCA86100A, Agilent Technologies Inc.) acquired the received timedomain response. The center wavelength of the DFB laser was $1310.3 \mathrm{~nm}$ with a pulse repetition rate of $10 \mathrm{MHz}$. In both methods, the back-to-back measurements were performed separately to obtain the system characteristic while no FUT was in place.

A 550-m long MMF of core diameter $62.5 \mu \mathrm{m}$ was used as the FUT. The impulse response measured with the system described in Fig. 2(c) showed the intuitive picture of the intermodal delays. Figure 3 shows the acquired impulse response of the MMF (solid line), displayed with the system response measured in the back-to-back condition (dotted line). The measured pulse width of the system response was $55 \mathrm{ps}$ in full width at half maximum (FWHM) for the dotted line of Fig. 3. In the impulse response of the MMF, the clearly observed two peaks suggested that the modal delays were distinguished by two mode groups while each group was dispersed slightly. The Fourier transforms of the responses gave the transfer function of $H(\omega)=F_{\text {out }} / F_{\text {in }}$ as suggested by Eq. (4) where $F_{\text {out }}$ and $F_{\text {in }}$ are the Fourier transforms of the impulse response and back-to-back system response, respectively.

Our ASE-based measurement system described in Fig. 2(b) directly acquired the power spectrum with the spectrum analyzer. The same FUT was used while the optical power incident onto the FUT was adjusted to 0.5 $\mathrm{mW}$. Figure 4 shows the screen-captured image of the electric spectrum analyzer with three traces: the noise level acquired with no optical input (A), the spectrum measured while the FUT in place (B), and the spectrum without the FUT in the back-to-back condition (C). The decrease of the spectrum $\mathrm{C}$ was caused by the limited bandwidth of the photoreceiver which affected commonly both of the

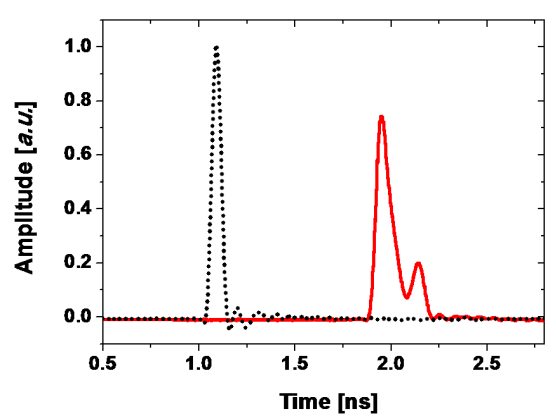

FIG. 3. Acquired impulse response of the MMF (solid line), displayed with the system response measured in the backto-back condition (dotted line). photoelectric spectra of B and C. Note that the acquired spectral data were all on the power of the photocurrent or the fourth-order power of the light fields. The optical intensity spectrum could be obtained by taking the square root of the acquired electric power. The transfer function could be obtained in this way. Instead, for minimization of the noise effect, an alternative formula was used which is given as

$$
H(\omega)=\left(\frac{G_{\text {out }}(\omega)-G_{\text {noise }}(\omega)}{G_{\text {in }}(\omega)-G_{\text {noise }}(\omega)}\right)^{1 / 2}
$$

where $G_{\text {out }}, G_{\text {in }}$ and $G_{\text {noise }}$ are the acquired power spectra of the FUT response, the back-to-back response and the noise, respectively. Because the incoherent random noise of $G_{\text {noise }}$ was added as an electric noise power to the spectra, the noise spectrum was subtracted before taking the square root in Eq. (11). This enhanced the measurement accuracy for a given power of the ASE.

Figure 5 shows the normalized transfer functions measured by the two methods of our ASE-base measurement (solid line) and the impulse response measurement (dotted line) for the 550-m MMF sample. Each was normalized so that the amplitude at frequency zero or the vicinity became a

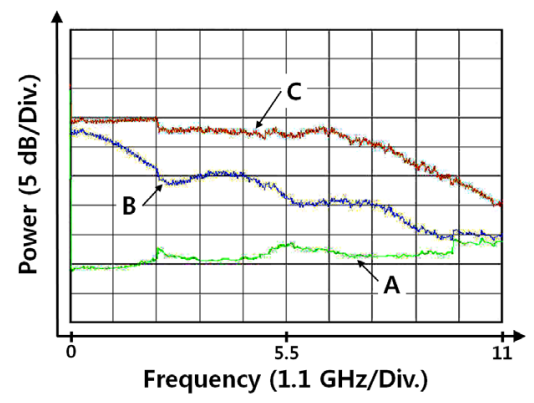

FIG. 4. Screen-captured image of the electric spectrum analyzer with three traces: the noise level acquired with no optical input (A), the spectrum measured while the FUT in place (B), and the spectrum without the FUT in the back-to-back condition (C).

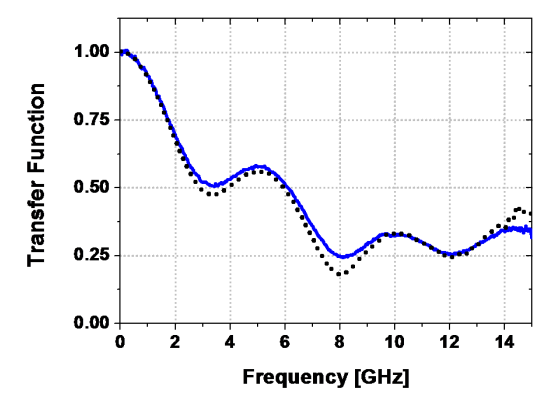

FIG. 5. Normalized transfer functions measured by the two methods of our ASE-base measurement (solid line) and the impulse response measurement (dotted line) for the 550-m MMF sample. 
unity, i.e., $H(0)=1$. Those two transfer functions showed very similar features. The cosine-like fluctuations must have come from the presence of the two mode groups that formed the distinct peaks in the impulse response observed in Fig. 3. The interval of the fluctuation was $\sim 5 \mathrm{GHz}$ as seen in Fig. 5 which well matched the separation of the impulse response peaks, $\sim 0.2 \mathrm{~ns}$, found in Fig. 3. The 3-dB bandwidth was difficult to determine with a certain value because of the fluctuating feature of the transfer function but could be estimated to be $\sim 3 \mathrm{GHz}$ by the first point that crossed or nearly crossed the discrimination level of $-3 \mathrm{~dB}(50 \%)$. The bandwidth-length product was estimated to be $\sim 3 \mathrm{GHz}$ by $0.55 \mathrm{~km}$ or $\sim 1.65 \mathrm{GHz} \cdot \mathrm{km}$ for the MMF sample.

The measurement repeatability was tested for the two methods. From the data obtained by five successive measurements performed in the same condition, a statistical deviation of the measured transfer functions was evaluated for each method. The interval between the consecutive measurements was $\sim 60$ seconds. A single back-to-back measurement was performed to obtain $F_{i n}$ or $G_{i n}$ in each case, which was used for calculation of the transfer functions. In the impulse response measurement system, each time-domain impulse response was acquired with a waveform average number of $N=16$ while the back-to-back response was acquired with $N=256$ for reduced noise. In our ASE-based measurement system, each spectrum trace was acquired with a sweep time of $1 \mathrm{~s}$ while the system characteristics including the noise spectrum were acquired with a longer acquisition time of $25 \mathrm{~s}$. Note that the back-to-back response of our ASE-based method was dominantly made by characteristics of the photoreceiver and the spectrum analyzer and does not need to be frequently calibrated.

Figure 6 shows the spectral standard deviation of the repeated measurements for the case of the ASE-based system (solid line) and that of the impulse response measurement system (dotted line). Spending similar acquisition times, our ASE-based measurement system exhibited significantly lower deviations especially for the high-frequency region above $10 \mathrm{GHz}$. The rapid increase of the error in the impulse response measurement could be explained by the limited bandwidth of the system response. Figure 7 shows the spectrum of the system response, $F_{\text {in }}$ (solid line) and its $-1 / 2^{\prime}$ th power, $F_{\text {in }}{ }^{-1 / 2}$ (dotted line) for the back-to-back system response measurement. The dotted curve in Fig. 7 showed a similar characteristic to that of the standard deviation of the impulse response measurement shown with the dotted line of Fig. 6. This suggested that the error of the transfer function measurement is inversely proportional to the square root of the spectral amplitude. Hence, the limited modulation bandwidth of the pulsed source was understood to result in considerable inaccuracy in the transfer function measurement.

By using our ASE-based system, measurements of a short fiber section were also performed with a 100-m MMF that originated from the same fiber spool. Figure 8 shows the normalized transfer functions obtained by five successive measurements for the 100-m MMF. Very small deviations were observed over the whole frequency range up to $15 \mathrm{GHz}$. The $3-\mathrm{dB}$ bandwidth was evaluated to be $15 \mathrm{GHz}$ or slightly higher, which makes a bandwidthlength product of $\sim 1.5 \mathrm{GHz} \cdot \mathrm{km}$ for the $0.1-\mathrm{km}$ section of the MMF. This agreed with the measurement result of the $550-\mathrm{m} \mathrm{MMF}, \sim 1.65 \mathrm{GHz} \cdot \mathrm{km}$. The difference of $10 \%$ was accounted for the intermodal coupling effect of the tightly spooled MMF in part [4]. The stable measurement result obtained by our method with the short MMF section demonstrated the feasibility of our measurement scheme in the field test where $>10 \mathrm{GHz}$ is needed for the measurement bandwidth.

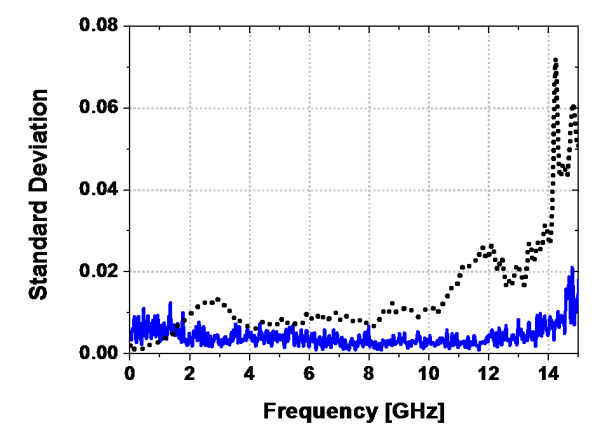

FIG. 6. Spectral standard deviation of the repeated measurements for the case of the ASE-based system (solid line) and that of the impulse response measurement system (dotted line).

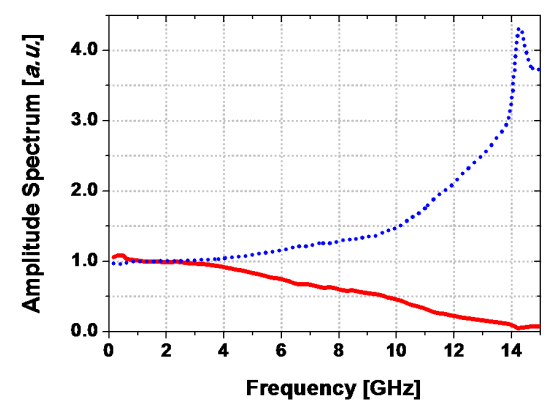

FIG. 7. Spectrum of the system response, $F_{\text {in }}$ (solid line) and its $-1 / 2$ 'th power, $F_{\text {in }}{ }^{-1 / 2}$ (dotted line) for the back-to-back system response measurement.

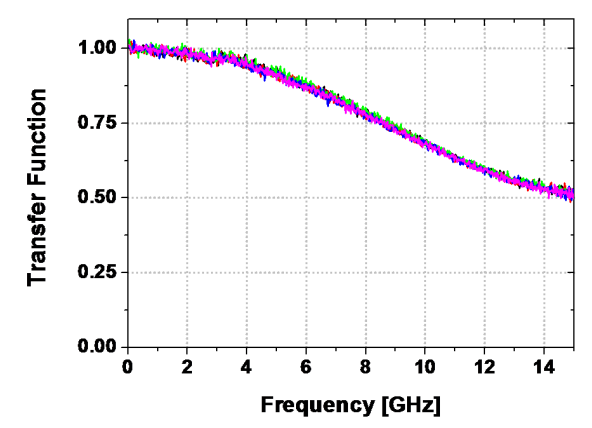

FIG. 8. Normalized transfer functions obtained by five successive measurements for the 100-m MMF. 


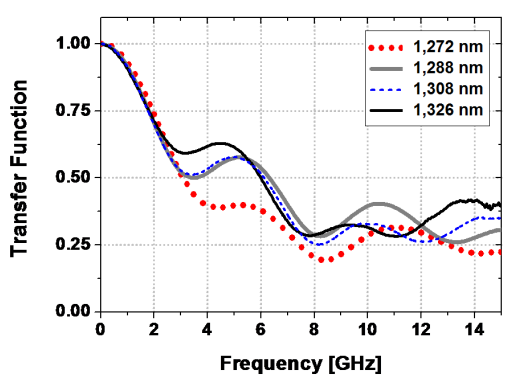

FIG. 9. Normalized transfer functions measured with the 550-m MMF sample at different wavelengths of 1,272 nm, $1,288 \mathrm{~nm}, 1,308 \mathrm{~nm}$ and 1,326 $\mathrm{nm}$, respectively.

One more advantage of our ASE-based MMF characterization scheme is found in its wide wavelength adjustability. Due to the wide optical bandwidth of the ASE source spectrum, a wavelength range of $\sim 50 \mathrm{~nm}$ can be covered by a single SOA light source, which can easily extended further with multiple SOAs or super-luminescent diodes. The adjustability can be obtained with ease by changing the center wavelength of the optical filter used in the source part. We measured the wavelength dependence of the MMF bandwidth by utilizing four different BPFs with a nominal wavelength spacing of $20 \mathrm{~nm}$. Figure 9 shows the normalized transfer functions measured with the 550-m MMF sample at different wavelengths of $1,272 \mathrm{~nm}$, $1,288 \mathrm{~nm}, 1,308 \mathrm{~nm}$ and $1,326 \mathrm{~nm}$. Here, the wavelength was evaluated by the mean wavelength of the filtered ASE spectrum. The experimental result showed that the transfer characteristic did not vary considerably for the lowfrequency band below $2 \mathrm{GHz}$ but, in contrast, it exhibited noticeable wavelength dependence in the high-frequency range. This feature of our ASE-based measurement scheme must be useful when the wavelength dependence of the bandwidth matters in the applications.

\section{DISCUSSION}

In this research, we showed that our MMF bandwidth measurement scheme has several advantages compared to the conventional measurement techniques. Our method utilizes a low-cost ASE light source without any modulation electronics, which greatly simplifies the measurement system and improves the measurement reliability. The measurement bandwidth can be easily extended to tens of $\mathrm{GHz}$ or higher owing to the wide bandwidth of the ASE light and the availability of the wideband electric spectrum analyzer that easily covers $40 \mathrm{GHz}$ or wider. The detected spectral density of the fourth-order power spectra can be further improved by reducing the optical bandwidth for a given total optical power. Then, with a higher signal-to-noise ratio, our system could measure the back-reflected responses of FUTs to double the measurement accuracy in a reflectometric configuration [12].

Our method has two unresolved issues that the present research has not revealed yet, and may need further investigations in separate studies. The first issue is related to the chromatic dispersion effect in the measurement result. In finding the transfer functions, the effect of GVD was neglected for simplicity as the conventional MMF bandwidth measurement also does. It could be justified by the fact that the modal dispersion is usually far larger than the GVD for typical MMFs. An MMF mostly has an intermodal delay definitely larger than $100 \mathrm{ps} / \mathrm{km}$ while the chromatic dispersion can be as large as $-100 \mathrm{ps} / \mathrm{nm} / \mathrm{km}$ at the short wavelength band of $850 \mathrm{~nm}$ [1]. At the $1,310-\mathrm{nm}$ band, it is surely negligible, below $5 \mathrm{ps} / \mathrm{nm} / \mathrm{km}$. But, in some cases, the measured MMF bandwidth can be considerably contaminated by the GVD effect that should be minimized or compensated [1,3]. At least, a rough estimation on the GVD effect should be provided for the ASE field that is obtained through an MMF.

The second issue of our ASE-based method is related to the absence of the phase information in our spectrum measurement. Our ASE-based measurement system detects the amplitude of the transfer function in losing the phase information that is essential for reconstructing the temporal contents of the impulse response via the inverse Fourier transform. In this context, our measurement method is unable to measure the intermodal delays but evaluates the bandwidth only, given in the frequency domain. This limitation may be a critical point of our measurement method because of the importance of the current laseroptimized MMF class for high-speed communications.

It has been found that the laser-irradiated MMF exhibits considerably different performances in actual communication systems from what the OFL bandwidth predicts [6]. The launch condition of the laser light usually makes a restricted mode launch (RML) without a mode scrambling mechanism. The differential modal delay (DMD) has been introduced for measuring the modal delays by differentiating the launch condition. The most well established way of differentiation is to translate the launching beam spot across the end-face of the MMF sample while the probe light is incident through the small beam onto the MMF e.g. by utilizing a scanned SMF launch. The obtained DMD data can be used to calculate the effective modal bandwidth (EMB or EMBc) with a set of the weightings that simulate certain types of the transmitters. Here, the last letter of 'c' in EMBc stands for 'calculated' when it is estimated from the DMD data. In the standard characterization method [6], the minimum value of the $\mathrm{EMBc}$ (i.e. minEMBc) stands for the bandwidth performance for guaranteed performance. The value of minEMBc is determined with the ten standard sets of weighting functions. Those rather complicated and time-consuming evaluation methods reflect the complexity of the MMF behavior with a non-specific transmitter. For the DMD or EMBc measurements, the complete information of the modal delays is required for further processing of the data.

To cope with the EMBc standard, we suggest three 
possible ways of utilizing our ASE-based measurement method for the high-speed MMF links with a particular emphasis on the field testing. The simplest way is to use our ASE-based OFL measurement as a rough estimator for the modal delays in an RML condition. The OFL bandwidth, itself, can still give useful information to compare the MMF installed in the field with the manufacturer's data. The careful analysis on the cosine fluctuations observed in the transfer function, if any, may give further information that is useful for estimating the actual link performance.

The second way is to introduce an optical mask for simulating a certain type of transmitters. This mask can be inserted between the ASE-irradiating SMF and the FUT and forms an incident field similar to the field distribution of the laser transmitter. An electrically controlled spatial light modulator (SLM) can be used for such a selective mode excitation with a capability of rapidly changing the weighting functions for a direct EMB measurement [13]. This approach can be highly preferable method in the field test, especially when the characteristics of the transmitters to be used are known. The third possible way is to develop an ASE-based measurement system that can measure the phase contents that will allow us to obtain the DMD data through the inverse Fourier transforms. Adding a separate reference arm that delivers the reference light of ASE in an SMF can be considered as in an MZI configuration $[8,10,11]$. But this approach increases the system complexity and may make our ASE-based method less attractive for the field testing.

\section{CONCLUSION}

In this research on the MMF bandwidth measurement, we introduced a novel MMF measurement scheme based on an ASE light source as a self-modulated light source. Through the experimental studies, we have found that our ASE-based method provides a wide measurement bandwidth with a small variation of the source characteristic when compared to the conventional impulse response measurement method. In our measurement system, the compactness of the source part which consists of passive fiber-optic components reduces the system complexity. In the field testing, the source part could be separated from the detection part being located at the other end of the FUT because of the non-synchronous nature of the measurement. The wavelength adjustability was also found to be an attractive feature of our method for the evaluation of wavelength-dependent MMF bandwidths. Due to those advantageous features, our ASE-based MMF bandwidth measurement scheme can be a promising alternative to the conventional pulse-based techniques. It is worth noting that the $850-\mathrm{nm}$ band is the major transmission window for the dominant high-speed MMF links. Further investigations on the 850-nm ASE-based measurement can enable full-band characterization of the MMF transmission capability.

\section{ACKNOWLEDGMENT}

This work has been supported in part by the Ministry of Education Science Technology (MEST) through the National Research Foundation of Korea (Grant No. 012R1A4A 102906) and by the Institute of Physics and Applied Physics at Yonsei University through BK21plus program.

\section{REFERENCES}

1. D. Molin, M. Bigot-Astruc, G. Kuyt, G. Mélin, and P. Sillard, "Multimode fibers for cost-effective high-speed, shortrange networks," in European Conference and Exhibition on Optical Communication (Amsterdam, Netherlands, 2012), paper Th.1.B.3.

2. TIA Specification for 850-nm Laser Optimized OM4 MMF, TIA-492AAAD (2009).

3. J. M. Castro, R. Pimpinella, B. Kose, and B. Lane, "Investigation of the interaction of modal and chromatic dispersion in VCSELMMF channels," J. Lightwave Technol. 30, 2532-2541 (2012).

4. J. E. Midwinter, Optical Fibers for Transmission (Krieger Publishing Company, Malabar, USA, 1992).

5. C. M. Miller, "Intensity modulation and noise characterization of optical signals," in Fiber Optic Test and Measurement, D. Derickson, ed. (Prentice Hall, Upper Saddle River, USA, 1998), pp. 246-283.

6. R. Ellis, "The importance of minEMBc laser bandwidth measured multimode fiber for high performance premises networks, White Paper WP1150 (Corning Inc., Corning, USA, 2007), http://www.corning.com/WorkArea/linkit.aspx?LinkIdentifier $=\mathrm{id} \&$ ItemID $=7881$.

7. M. B. Shemirani, W. Mao, R. A. Panicker, and J. M. Kahn, "Principal modes in graded-index multimode fiber in presence of spatial- and polarization-mode coupling," J. Lightwave Technol. 27, 1248-1261 (2009).

8. S. Moon, H. Noh, and D. Y. Kim, "Frequency-domain acquisition of fourth-order correlation by spectral intensity interferometry," Opt. Express 21, $23206-23219$ (2013).

9. T.-J. Ahn, S. Moon, Y. Youk, Y. Jung, K. Oh, and D. Y. Kim, "Mode analysis and modal delay measurement of a few-mode fiber by using optical frequency domain reflectometry," J. Opt. Soc. Korea 9, 54-58 (2005).

10. T.-J. Ahn, S. Moon, S. Kim, K. Oh, D. Y. Kim, J. Kobelke, K. Schuster, and J. Kirchhof, "Frequency-domain intermodal interferometer for the bandwidth measurement of a multimode fiber," Appl. Opt. 45, 8238-8243 (2006).

11. J. Y. Lee, T.-J. Ahn, S. Moon, Y. C. Youk, Y. M. Jung, K. Oh, and D. Y. Kim, "Fourier-domain low-coherence interferometry for differential mode delay analysis of an optical fiber," Opt. Lett. 31, 2396-2398 (2006).

12. S. Moon and D. Y. Kim, "Reflectometric fiber dispersion measurement using a supercontinuum pulse source," IEEE Photon. Technol. Lett. 21, 1262-1264 (2009).

13. J. Carpenter and T. D. Wilkinson, "Characterization of multimode fiber by selective mode excitation," J. Lightwave Technol. 30, 1386-1392 (2012). 